\title{
Prevalencia de hábitos orales y sus consecuencias dentomaxilares en escolares
}

\section{Oral habits prevalence and their dental-maxillary consequences in school children}

\author{
Cristina Mercedes Crespo Crespo ${ }^{1}$, Julieta Guadalupe Carrasco Aulestia ${ }^{1}$, Mariela Del Carmen Ramirez \\ Velásquez $^{1}$ y Henry Jonathan Chicaiza Sánchez ${ }^{1}$ \\ ${ }^{1}$ Universidad Católica de Cuenca, Ecuador \\ *cmcrespoc@ucacue.edu.ec
}

DOI: https://doi.org/10.26871/killkana_salud.v4i2.330

\begin{abstract}
Resumen
Contexto: Las alteraciones dentomaxilares pueden ser causada por diversos factores genéticos y/o ambientales, los más conocidos son los hábitos bucales que pueden llegar a afectar el crecimiento y desarrollo cráneo facial. Objetivo: El objetivo de esta investigación fue determinar la prevalencia de los hábitos de respiración bucal, succión digital y deglución atípica y sus consecuencias dentomaxilares, en escolares de 6 a 11 años de edad, de la escuela Emilio Abad de la ciudad de Azogues, Ecuador. Metodología: Se realizó una investigación observacional de tipo transversal y descriptiva, aplicada a un total de 185 niños en dos etapas; la primera consistió en un examen clínico y luego la aplicación de una encuesta, para obtener tablas de frecuencias, y la interpretación adecuada de las variables del estudio, relacionando las mismas mediante la prueba de Chi cuadrado con un nivel de significancia de 0.05. Resultados: Los resultados mostraron que el $77 \%$ de la muestra consistió en escolares masculinos y el $78 \%$ femenino, se evidenció la prevalencia de hábitos bucales en los niños evaluados, donde un $77.29 \%$ presentó hábitos de respiración bucal, succión digital y deglución atípica; siendo mayormente afectados la población en edades comprendidas entre 8 y 9 años con un $39.86 \%$. Conclusiones: El hábito de respiración bucal fue el más frecuente con un $26 \%$, y el menos predominante la succión digital con un $19 \%$. Es evidente, según los resultados, que todos los hábitos bucales reflejaron un porcentaje de consecuencias dentomaxilares, donde la respiración bucal y la mordida abierta fueron las de mayor prevalencia en los escolares de la Escuela Emilio Abad.
\end{abstract}

Palabras clave: hábitos bucales, maloclusiones, prevalencia. consecuencias dentomaxilares.

\begin{abstract}
Context: Dentomaxillary alterations can be caused by various genetic and/or environmental factors; the best known are oral habits that can affect cranial-facial growth and development. Objective: The objective of this research was to determine oral breathing, digital suction and atypical swallowing habits' prevalence, as well as their dental-maxillary consequences, in schoolchildren aged 6 to 11 years old, attending Emilio Abad School in the city of Azogues, Ecuador. Methodology: A descriptive cross-sectional and observational research was conducted, applied to 185 children, in two stages; the first one consisted on a clinical examination, and then a survey was administered, to obtain frequency tables, and the adequate interpretation of the study variables; relating them employing the Chi-square test with a 0.05 significance level. Results: The results showed that $77 \%$ of the sample were male school children, and $78 \%$ were girls. $77.29 \%$ of them presented oral breathing habits, digital suction and atypical swallowing; being the population aged between 8 and 9 years, the most affected, with $39.86 \%$. Conclusions: Mouth breathing, was the most frequent habit with $26 \%$, while digital suction was the least predominant with 19\%. According to the results, it is evident that all oral habits revealed a percentage of dental-maxillary consequences, being mouth breathing and an open bite, the most prevalent in Emilio Abad School students.
\end{abstract}

Keywords: Oral habits, malocclusions, prevalence, dental-maxillary consequences.

\section{Introducción}

Las maloclusiones, derivadas en muchos casos de hábitos orales, según la Organización Mundial de la Salud (OMS), ocupan el tercer lugar de prevalencia dentro de los problemas de salud bucodental, luego de la caries dental y de la enfermedad periodontal. ${ }^{1}$ La mayoría de las enfermedades bucales y en particular las maloclusiones, no ponen en riesgo la vida del paciente, pero su prevalencia e incidencia, son consideradas un problema de salud pública. ${ }^{2-4}$ 
Las alteraciones dentomaxilares pueden ser causada por diversos factores genéticos y/o ambientales, los más conocidos hábitos bucales que pueden llegar a afectar los tejidos blandos y alterar el crecimiento y desarrollo craniofacial. ${ }^{2}$ Los hábitos bucales son esquemas neuromusculares aprendidos, definidos también como una costumbre que se adquiere de un acto, inicia como un acto voluntario o consciente, y posteriormente se convierte en involuntario o inconsciente. $^{5-7}$

Los hábitos más frecuentes son; la respiración bucal, succión digital, deglución atípica. ${ }^{8}$ En el caso de la respiración bucal, el aire pasa por la cavidad oral, provocando un aumento de la presión aérea oral produciendo el moldeado y profundización del paladar, al no ocurrir el pasaje normal del aire por la cavidad nasal provoca la penetración de aire en los senos maxilares tornándolos atrésicos, y dando al paciente un aspecto característico de cara larga o fascie adenoidea; su etiología obstrucción funcional o anatómica, presencia de adenoides hipertróficas, cornetes hipertróficos, tabique desviado, inflamación de la mucosa por infecciones o alergias. ${ }^{9,10}$

La succión digital, afecta la estética y la función, protruyendo los incisivos centrales superiores, retrayendo los inferiores y en muchos casos origina mordida abierta que pueden llegar hasta el colapso maxilar y en consecuencia mordida cruzada posterior. Las repercusiones de este hábito derivan de la posición baja de la lengua, que deja ejercer presión sobre el paladar, de la hiperactividad de los músculos buccinadores que tienden a comprimir el paladar, de la presión pasiva del dedo sobre las arcadas dentarias y de la fuerza que ejerce el dedo sobre el paladar. ${ }^{11,12}$

La deglución atípica o interposición lingual, es producido por persistencia del patrón de deglución infantil posterior a la erupción de los dientes anterior, se trata de un fenómeno secundario a la presencia de una mordida abierta anterior. Su etiología depende de la alimentación artificial por medio del biberón, amígdalas inflamadas, desequilibrio del control nervioso macroglosia, anquiloglosia, perdida temprana de los dientes anteriores, presencia de un diastema interincisal grande, etc. ${ }^{12,13}$

El daño provocado por los hábitos depende de varios factores, la edad en la que empieza el hábito por lo que a menor edad el daño será mayor, relacionado esto además con el tiempo de duración, la frecuencia y la intensidad con el cual se practica el mismo. ${ }^{14}$

El objetivo del estudio fue determinar la prevalencia de respiración bucal, succión digital y deglución atípica así como sus consecuencias dentomaxilares en escolares de 6 a 11 años de edad.

\section{MaterialesyY Método}

\subsection{Diseño de estudio}

Se realizó una investigación descriptiva, observacional, trasversal, a través de un cuestionario de preguntas para la obtención de la información en relación a hábitos bucales presentes o pasados y un examen clínico, el cual consistió en exploración facial e intraoral,

\subsection{Población y Muestra}

La población estuvo constituida de 700 niños entre 6 y 11 años de edad, pertenecientes a la unidad educativa Emilio Abad de la ciudad de Azogues Ecuador, localizado en la zona urbana, seleccionados estos aplicando los siguientes criterios de inclusión: estudiantes cuyos padres firmen el consentimiento, además de que acepten ser parte de la investigación mediante asentimiento informado, luego de explicarles el propósito del estudio; excluyendo a quienes sean o hayan sido portadores de aparatología ortopédica u ortodoncica; obteniendo así una muestra de 185 escolares de ambos sexos, para evaluar sus hábitos orales y sus manifestaciones bucales.

\subsection{Método}

Esta investigación se realizó en dos etapas, la primera consistió en un examen clínico a los escolares participantes y luego la aplicación de la encuesta.

\section{Examen clínico}

Previo a la encuesta (figura 1), se llevó a cabo el examen clínico, que consistió en la exploración tanto física como intraoral, con las debidas normas de bioseguridad con el uso de gorro, guantes, mascarillas, baja lenguas desechables; durante el mismo se le solicitó al niño abrir la boca para observar la presencia de alguna características dentomaxilar como mordida abierta, paladar profundo, vestibularización, labio hipertónico, gingivitis y apiñamiento, lo cual se registró en el formulario (figura 1) así como los hallazgos relacionados con la presencia de hábitos de respiración bucal, succión digital o deglución atípica.

\section{Aplicación de la encuesta}

Para obtener los datos sobre hábitos orales presentes o pasados de los participantes del estudio, se aplicó la encuesta a cada uno de los adolescentes, el cual consistió en una serie de peguntas, cuyas respuestas fueron registradas en el mismo. (figura 1),

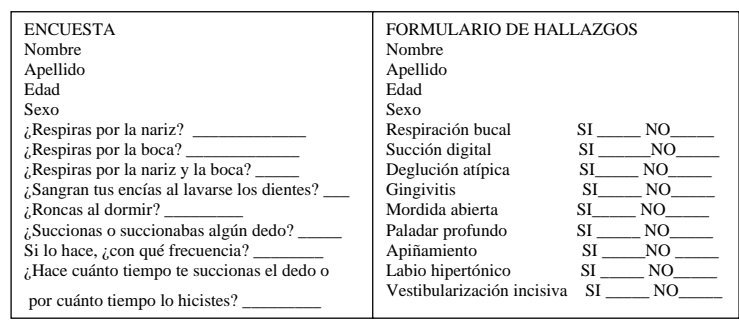

Fig. 1: Encuesta y formulario

Fuente: Propia 


\section{Análisis estadístico de los resultados}

Luego de obtenida la base de datos en archivo de Excel, el análisis estadístico se llevó a cabo a través del paquete estadístico SPSS Statistics 24, Haciendo uso de la estadística descriptiva se obtuvieron tablas de frecuencias, las cuales permitieron la interpretación adecuada del conjunto de variables que componían el estudio. Seguidamente para comparar la distribución observada de los datos con una distribución esperada, estableciendo la relación entre estas variables se empleó la prueba de Chi cuadrado con un nivel de significancia estadística de 0,05 .

\section{Resultados}

Esta investigación muestra los resultados obtenidos a través de la inspección clínica y el cuestionario aplicado a un total de 185 escolares con edades comprendidas entre 6 y 11 años de edad de la escuela Emilio Abad de la Ciudad de Azogues para determinar la prevalencia de hábitos orales y sus consecuencias dentomaxilares.

Los resultados de la tabla 1 muestran la prevalencia de hábitos bucales en los niños evaluados, donde un 77 . $29 \%$ de las distintas edades evidenció presencia de hábitos de respiración bucal, succión digital y deglución atípica; siendo mayormente afectados la población en edades comprendidas de 8-9 años con un 39.86\% del total de los 143 adolescentes que presentaron los hábitos estudiados., El hábito de respiración bucal fue el más frecuente con un $44 \%$. Los resultados obtenidos no mostraron significancia estadística.

Tabla 1: Prevalencia de respiración bucal, succión y deglución atípica en la escuela Emilio Abad de la ciudad de Azogues según grupos de edad

\begin{tabular}{|c|c|c|c|c|c|c|}
\hline \multirow{2}{*}{ Edad } & \multicolumn{2}{|c|}{ Presencia } & \multicolumn{2}{|c|}{ Ausencia } & \multirow{2}{*}{ Total } & \multirow[b]{2}{*}{$\mathrm{p}^{*}$} \\
\hline & $\mathrm{N}$ & $\%$ & $\mathrm{~N}$ & $\%$ & & \\
\hline 6 a 7 & 55 & 77 & 20 & 27 & 75 & 0,624 \\
\hline 8 a 9 & 57 & 84 & 11 & 16 & 68 & \\
\hline 10 a 11 & 31 & 73 & 11 & 27 & 42 & \\
\hline Total & 143 & & 42 & & 185 & \\
\hline
\end{tabular}

Fuente: Fuente propia.

N: Frecuencia absoluta. \%: Frecuencia relativa. p: Significancia estadística. *: Prueba de Chi-cuadrado

Tabla 2: Prevalencia de respiración bucal, succión y deglución atípica en la escuela Emilio Abad de la ciudad de Azogues según sexo

\begin{tabular}{ccccccc}
\hline \multirow{2}{*}{ Sexo } & \multicolumn{2}{c}{ Presencia } & \multicolumn{2}{c}{ Ausencia } & Total & \multirow{2}{*}{$\mathrm{p}^{*}$} \\
\cline { 2 - 5 } & $\mathrm{N}$ & $1 \%$ & $\mathrm{~N}$ & $1 \%$ & número & 0.973 \\
\hline Masculino & 72 & 77 & 22 & 23 & 94 & 0,913 \\
Femenino & 71 & 78 & 20 & 22 & 91 & \\
Total & 143 & & 42 & & 185 & \\
\hline
\end{tabular}

Fuente: Fuente propia.

N: Frecuencia absoluta. \%: Frecuencia relativa. p: Significancia estadística. *: Prueba de Chi-cuadrado

Los resultados de la tabla 2 muestran la prevalencia de hábitos bucales en los escolares evaluados en ambos sexos, donde se observa que la muestra fue homogénea, Se encontró que la respiración oral fue el hábito bucal más predominante para ambos sexos $(26 \%)$, y el menos predomínate la succión digital (19\%).

Tabla 3: Consecuencias dentomaxilares de respiración bucal en niños de 6-11 años de edad de la escuela Emilio Abad de la ciudad de Azogues en el período 2016

\begin{tabular}{cccccc}
\hline \multirow{2}{*}{ Alteraciones } & \multicolumn{2}{c}{ Presencia } & \multicolumn{2}{c}{ Ausencia } & \multirow{2}{*}{ Total } \\
\cline { 2 - 4 } & $\mathrm{N}$ & $\%$ & $\mathrm{~N}$ & $\%$ & \\
\hline Mordida abierta & 54 & $86 \%$ & 9 & $14 \%$ & 63 \\
Paladar profundo & 45 & $71 \%$ & 18 & $29 \%$ & 63 \\
Vestibularización incisiva & 25 & $40 \%$ & 38 & $60 \%$ & 63 \\
Labio hipertónico & 0 & $0 \%$ & 63 & $100 \%$ & 63 \\
Gingivitis & 28 & $44 \%$ & 35 & $56 \%$ & 63 \\
Apiñamiento & 24 & $38 \%$ & 39 & $62 \%$ & 63 \\
\hline
\end{tabular}

Fuente: Fuente propia.

N: Frecuencia absoluta. \%: Frecuencia relativa. p: Significancia estadística. *: Prueba de Chi-cuadrado

La tabla 3 evidencia que de los 185 adolescentes investigados solo 63 casos lo cual representa el $44.05 \%$ de la muestra estudiada presentó, características dentomaxilares como consecuencia del hábito de respiración bucal. Siendo de mayor presencia la mordida abierta $(86 \%)$, paladar profundo $(71 \%)$, gingivitis $(44 \%)$, vestibularizacion incisiva (40\%). Cabe acotar que del $63 \%$ de los adolescentes con habito de respiración bucal solo el labio hipertónico, no se presentó como manifestación característica.

Tabla 4: Consecuencias dentomaxilares de succión digital en niños de 6-11 años de edad de la escuela Emilio Abad de la ciudad de Azogues en el período 2016

\begin{tabular}{cccccc}
\hline \multirow{2}{*}{ Alteraciones } & \multicolumn{2}{c}{ Presencia } & \multicolumn{2}{c}{ Ausencia } & \multirow{2}{*}{ Total } \\
\cline { 2 - 4 } & $\mathrm{N}$ & $\%$ & $\mathrm{~N}$ & $\%$ & \\
\hline Mordida abierta & 21 & $58 \%$ & 15 & $42 \%$ & 36 \\
Paladar profundo & 1 & $3 \%$ & 35 & $97 \%$ & 36 \\
Vestibularización incisiva & 23 & $64 \%$ & 13 & $36 \%$ & 36 \\
Labio hipertónico & 0 & $0 \%$ & 36 & $100 \%$ & 36 \\
Gingivitis & 10 & $28 \%$ & 26 & $72 \%$ & 36 \\
Apiñamiento & 7 & $19 \%$ & 29 & $81 \%$ & 36 \\
\hline
\end{tabular}

Fuente: Fuente propia.

N: Frecuencia absoluta. \%: Frecuencia relativa. p: Significancia estadística. *:

Prueba de Chi-cuadrado

Del total de la muestra solo 36 adolescentes presentó el hábito de succión digital lo cual representó el $25.17 \%$. Las evidencias muestran que al igual que en el hábito de succión digital el labio hipertónico fue la característica no encontrada en los adolescentes estudiados. Las consecuencias dentomaxilares del hábito de succión digital en orden de aparición de frecuencia fue: vestibularización incisiva en un $64 \%$, seguida de mordida abierta y gingivitis con un $58 \%$ y $28 \%$ respectivamente. (Tabla 4 ) 
Tabla 5: Consecuencias dentomaxilares de deglución atípica en niños de 6-11 años de edad de la escuela Emilio Abad de la ciudad de Azogues en el período 2016

\begin{tabular}{cccccc}
\hline \multirow{2}{*}{ Alteraciones } & \multicolumn{2}{c}{ Presencia } & \multicolumn{2}{c}{ Ausencia } & \multirow{2}{*}{ Total } \\
\cline { 2 - 5 } & $\mathrm{N}$ & $\%$ & $\mathrm{~N}$ & $\%$ & \\
\hline Mordida abierta & 23 & $52 \%$ & 21 & $48 \%$ & 44 \\
Paladar profundo & 0 & $0 \%$ & 44 & $100 \%$ & 44 \\
Vestibularización incisiva & 28 & $64 \%$ & 16 & $36 \%$ & 44 \\
Labio hipertónico & 44 & $100 \%$ & 0 & $0 \%$ & 44 \\
Gingivitis & 6 & $14 \%$ & 38 & $86 \%$ & 44 \\
Apiñamiento & 15 & $34 \%$ & 29 & $66 \%$ & 44 \\
\hline
\end{tabular}

Fuente: Fuente propia.

N: Frecuencia absoluta. \%: Frecuencia relativa. p: Significancia estadística. *: Prueba de Chi-cuadrado

El hábito de deglución atípica estuvo presente en un $30.76 \%$ de los adolescentes estudiados, observándose en los mismos, a diferencia de los hábitos descritos anteriormente como principal consecuencia dentomaxilar presente el labio hipertónico.

\section{Discusión}

Los resultados de esta investigación, realizada en la escuela Emilio Abad localizada en una zona urbana de la ciudad de Azogues Ecuador, en una muestra de 185 adolescentes, permitieron determinar la prevalencia de las maloclusiones y sus consecuencias dentomaxilares encontrándose rasgos característicos como mordida abierta, paladar profundo, vestibularizacion incisiva, gingivitis y apiñamiento.

A diferencia de lo reportado por Reyes Romagosa3 en su estudio, donde prevaleció la succión digital $(38.4 \%)$, el hábito de respiración bucal fue el de mayor prevalencia con el $44.05 \%$, de la población estudiada, diferenciándose además del estudio de Mendozal cuyo estudio evidenció una prevalencia menor de respiración bucal con un $31.8 \%$.

En el estudio realizado por Espinoza Calle ${ }^{13}$ y cols, en niños de 6 a 12 años para determinar la prevalencia de hábitos orales, la deglución atípica fue con un $19.48 \%$ la mayormente encontrada, seguida por el hábito de succión digital con un $2.19 \%$ y solo el $0.20 \%$ presentaron respiración bucal, mientras que en esta investigación se determinó la mayor prevalencia del hábito de respiración bucal seguido de la deglución atípica y la succión digital con $30.76 \%$ y $25,17 \%$ respectivamente.

El estudio de Medina4 reporta como consecuencias dentomaxilares mayormente encontradas la mordida abierta $31.5 \%$ y en segundo lugar el apiñamiento con un $19.6 \%$ a diferencia de esta investigación que muestra evidencia de mayor prevalencia de mordida abierta (86\%) como consecuencia de adolescentes respiradores bucales, siendo la característica mayormente encontrada la vestibularizacion de dientes anterosuperiores, lo cual es bien conocido que es generalmente una característica asociada en los casos de los hábitos estudiados

\section{Conclusión}

La mayor prevalencia de hábitos bucales fue respiración bucal, succión digital y deglución atípica siendo mayor en el grupo de escolares de 8-9 años, y en el sexo masculino.

La consecuencia dentomaxilar asociada a la respiración bucal de mayor frecuencia fue la mordida abierta; en el caso del hábito de succión digital la vestibularización incisiva y en la deglución atípica el labio hipertónico.

\section{Fuente de Financiamiento}

Este estudio es autofinanciado.

\section{Conflicto de Intereses}

No existen conflictos personales, profesionales, financieroso de otro tipo.

\section{Consentimiento Informado}

Los autores cuentan con el consentimiento informado de los pacientes para la investigación, la publicación del caso y sus imágenes.

\section{Referencias Bibliográficas}

1. Mendoza Oropeza L, Meléndez Ocampo AF, Ortiz Sánchez R, Fernández López A. Prevalencia de las maloclusiones asociada con hábitos bucales nocivos en una muestra de mexicanos. Medigraphic; 2014. Available from: http://revistas.unam.mx/index.php/rmo/article/ view/54211.

2. Lee Garcés Y, Lee Garcés Y, Ballart Ramos A, Simón Rodriguez J, Guerrero Silva I. Intervención educativa sobre hábitos bucales deformantes en escolares de 6 a 12 años. Revista Información Científica. 2015;93(5):11211131. Available from: http://www.revinfcientifica.sld.cu/ index.php/ric/article/view/182.

3. Reyes Romagosa DE, Torres Pérez I, Quesada Oliva LM, Milá Fernández M, Labrada Estrada HE. Hábitos bucales deformantes en niños de 5 a 11 años. MEDISAN. 2014 05;18:606 - 612. Available from: http://scielo.sld.cu/scielo.php?script=sci_arttext\&pid= S1029-30192014000500003\&nrm=iso.

4. Chamorro AF, García C, Mejía E, Viveros E, LLanos LS, Triana FE, et al. Hábitos orales frecuentes en pacientes del área de Odontopediatría de la Universidad del Valle. Revista CES Odontología. 2016;29(2):4. Available from: https://dialnet.unirioja.es/servlet/articulo? codigo $=5759180$.

5. Martín Zaldivar L, García Peláez S, Expósito Martín I, Estrada Verdeja V, Pérez Llanes Y. Deglución anormal: algunas consideraciones sobre este hábito. Revista Archivo Médico de Camagüey. 2010 12;14:1 - 10. Available from: http://scielo.sld.cu/scielo.php?script=sci_ arttext\&pid=S1025-02552010000600021\&nrm=iso.

6. García Molina GI. Etiología y Diagnóstico de pacientes Respiradores Bucales en edades tempranas-Revisión bibliográfica. Revista latinoamericana de Ortodoncia y 
Odontopediatría. 2011. Available from: https://www. 12. Fernández OC. Asociación de los malos hábitos orales ortodoncia.ws/publicaciones/2011/art-18.

7. Franco Varas V, Gorritxo Gil B, García Izquiercon las anomalías dentomaxilares. Redoe. 2015;12(29).

do F. Prevalencia de hábitos orales infantiles y 13. Espinoza CI, Casas AL, Campos CK. Prevalencia de su influencia en la dentición temporal. Pediatría Atención Primaria. 2012 03;14:13 - 20. Available from: http://scielo.isciii.es/scielo.php?script=sci_arttext\& pid $=$ S1139-76322012000100002\&nrm $=$ iso. hábitos orales y alteraciones dentoalveolares en niños de 6 a 12 años. Odontol pediatr. 2016;15(2):127-134. Available from: http://pesquisa.bvsalud.org/portal/resource/pt/ biblio-835086?lang=es.

8. Laboren M, Medina C, Viloria C, Quirós O, D’Jurisic A, 14. Aguilar Roldán M, Nieto Sánchez I, De la Cruz Pérez Alcedo $\mathrm{C}$, et al. Hábitos bucales más frecuentes y su relación con maloclusiones en niños con dentición primaria. Revista Latinoamericana de Ortodoncia y Odontopediatria. 2010. Available from: https://www.ortodoncia.ws/ publicaciones/2010/art-20/.

9. Silva Pérez GA, Bulnes López RM, Rodríguez López LV. Prevalencia de hábito de respiración oral como factor etiológico de maloclusión en escolares del Centro, Tabasco. Revista ADM. 2014;71(6).

Recibido: 10 de julio de 2019

10. Ricardo Reyes M. Comportamiento de escolares de primaria con hábito de succión digital luego de una intervención educativa. MEDISAN. 2011 02;15:222 - 227. Available from: http://scielo.sld.cu/scielo.php?script=sci_ arttext\&pid=S1029-30192011000200012\&nrm=iso.

11. González M, Guida G, Herrera D, Quirós O. Maloclusiones asociadas a: Hábito de succión digital, hábito de deglución infantil o atípica, hábito de respiración bucal, hábito de succión labial y hábito de postura. Rev Latinoam Ortod Odontopediatr. 2012. Available from: https://www. ortodoncia.ws/publicaciones/2012/art-28/. J. Relación entre hábitos nocivos y maloclusiones en una muestra de 525 pacientes de Ortodonia. Ortodonciaws. 2011. Available from: https://www.ortodoncia.ws/ publicaciones/2011/art-25/.

de abril de 2020 
